\title{
Lacrimal Gland Involvement in Blepharophimosis-Ptosis-Epicanthus Inversus Syndrome
}

Ana Filipa Duarte, MD, ${ }^{1}$ Patricia M.S. Akaishi, MD, ${ }^{1}$ Greice A. de Molfetta, PhD, ${ }^{2}$ Salomão Chodraui-Filho, MD, ${ }^{3}$ Murilo Cintra, MD, ${ }^{3}$ Alcina Toscano, MD, ${ }^{4}$ Wilson Araujo Silva, Jr., PhD, ${ }^{2}$ Antonio A.V. Cruz, $\mathrm{MD}^{1}$

Purpose: To describe the involvement of the lacrimal gland (LG) in blepharophimosis-ptosis-epicanthus inversus syndrome (BPES).

Design: Observational, cross-sectional study.

Participants: Twenty-one patients with BPES (10 female, 11 male) aged on average 15 years (range, 2-39 years), from 3 Brazilian medical centers and 1 Portuguese medical center.

Methods: Patients had their ocular surface evaluated with slit-lamp biomicroscopy, and tear production quantified with the Schirmer test I. The LG volumes were measured on computed tomography (CT) scans in the BPES sample and in a group of age-matched subjects imaged for nonorbital diseases. Sixteen patients were screened for mutations in the FOXL2 gene.

Main Outcome Measures: Lacrimal meniscus height, Schirmer test I, presence of superficial punctate keratopathy (SPK), LG volume, and molecular analysis of the FOXL2 gene.

Results: Absence of LG was detected bilaterally in 9 patients (42.8\%) and unilaterally in 2 patients $(9.5 \%)$. When considering only patients with measurable LG, the median volume was $0.22 \mathrm{~cm}^{3}$ in the right eye (range, $0.06-0.36 \mathrm{~cm}^{3}$ ) and $0.24 \mathrm{~cm}^{3}$ in the left eye (range, $0.08-0.34 \mathrm{~cm}^{3}$ ). These values were significantly lower than those for the age-matched controls (median $=0.54$ right eye and 0.53 left eye; $P<0.05$ ). There was a significant association between deficiency of tear production and LG volume reduction and agenesis. Molecular analysis of the FOXL2 gene revealed the presence of 8 distinct mutations, 4 of them novel ones. A significant reduction of LG size or agenesis was associated with mutations affecting protein size (due to underlying changes in the stop codon location) or the DNA-binding forkhead domain (Fisher exact test, $P=0.021$ ). In 3 probands, the underlying genetic defect was not found.

Conclusions: This is the first study reporting LG volumes in BPES, describing a significant number of patients with LG agenesis. The association between alacrima and BPES is not incidental, and a thorough evaluation of tear production is recommended especially if ptosis surgery is planned. Ophthalmology 2017;124:399-406 @ 2016 by the American Academy of Ophthalmology

Blepharophimosis-ptosis-epicanthus inversus syndrome (BPES) (Online Mendelian Inheritance in Man 110100) is an uncommon disorder first described in 1889 by Vignes. ${ }^{1}$ Three decades later, Dimitry ${ }^{2}$ defined its inheritance pattern as autosomal dominant, which was confirmed over subsequent years. Its molecular basis remained undetermined until 2001, when Crisponi et $\mathrm{al}^{3}$ finally identified forkhead box protein L2 (FOXL2) (Online Mendelian Inheritance in Man *605597) on chromosome $3 \mathrm{q} 23$ as the causal gene. ${ }^{3}$ This single-exon gene encodes a forkhead transcription factor expressed in the developing eyelid mesenchyme and fetal and adult ovaries. ${ }^{4,5}$ Thus, depending on its expression, there are 2 types of BPES: type I with premature ovarian failure and type II with normal ovary function. ${ }^{6,7}$ Severe ptosis with poor levator function, epicanthus inversus, and telecanthus are present in both types. We describe a significant spectrum of lacrimal gland (LG) changes in BPES. In addition, molecular analysis of
FOXL2 was performed in 16 patients in an attempt to obtain new insights into a possible genotype-phenotype correlation.

\section{Methods}

\section{Patients}

The research adhered to the tenets of the Declaration of Helsinki. Approval was obtained from the Institutional Review Boards and Ethics Committees, and all subjects gave written informed consent to participate in the study.

Twenty-one patients with a clinical diagnosis of BPES were recruited from 1 Portuguese and 3 Brazilian medical centers. Mean age was 15 years (range, 2-39 years). Schirmer test I (without anesthesia) and slit-lamp biomicroscopy assessment of the lacrimal meniscus height and corneal fluorescein staining were performed in all patients. A Schirmer's test score $<10 \mathrm{~mm}$ after 5 minutes with 
Table 1. Primers Used for Amplification and Sequencing of the Entire Coding Region of the FOXL2 Gene

\begin{tabular}{|c|c|c|c|}
\hline Primer Names & Primer Sequences $\left(5^{\prime}>3^{\prime}\right)$ & Size of the PCR Product (bp) & Annealing Temperature $\left({ }^{\circ} \mathrm{C}\right)$ \\
\hline FOXL2-1.1F & TTTGAGACTTGGCCGTAAGC & 444 & 55 \\
\hline FOXL2-1.1R & TGATGAAGCACTCGTTGAGG & & \\
\hline FOXL2-1.2F & CGAAGTTCCCGTTCTACGAG & 576 & 57 \\
\hline FOXL2-1.2R & CCAGGCCATTGTACGAGTTC & & \\
\hline FOXL2-1.3F & GTACACACGCGTGCAGAGC & 407 & 57 \\
\hline FOXL2-1.3R & CGTCCCTGCATCCTCGCATC & & \\
\hline
\end{tabular}

the eyes closed was considered as an indication of reduced aqueous tear production. ${ }^{8,9}$ Two radiologists measured LG volumes on computed tomography (CT) scans using OsiriX software (developed by Pixmeo, SARL, Geneva; http://www.osirix-viewer.com) as the Digital Imaging and Communications in Medicine (DICOM) viewer. Briefly, as described by Bingham et al, ${ }^{10}$ the LG area was measured from consecutive axial slices of orbital CT scans and added to obtain a final volume. The same protocol was applied to a group of 30 age-matched patients imaged for nonorbital pathology, which constituted the control group. The Wilcoxon signed-rank test was used to compare right and left LG volumes, and the Mann-Whitney test was used to compare LG volumes between groups. The Mann-Whitney $U$ test was used to compare the Schirmer's test scores between eyes with nonmeasurable versus reduced LG. Finally, the Fisher exact test was used to analyze the association between the signs of keratopathy and LG size categorized as reduced and nonmeasurable. In all these tests, a $P$ value $\leq 0.05$ was considered significant.

\section{DNA Amplification and Exon Sequencing}

Sixteen patients were submitted to exon sequencing of the FOXL2 gene. Genomic DNA was extracted from peripheral blood leukocytes using a QIAamp DNA Blood isolation kit (Qiagen, Hilden, Germany) according to the manufacturer's instructions. For the analysis of the FOXL2 gene, 3 pairs of primers were designed to cover the entire FOXL2 exon and its splice site junctions (Table 1). The polymerase chain reaction-amplified DNA fragments were subjected to direct sequencing using the automatic capillary sequencing system ABI 3500X Genetic Analyzer (Applied Biosystems, Foster City, CA) and the Big Dye terminator v3.1 cycle sequencing kit (Applied Biosystems) according to the manufacturer's instructions. The results were analyzed using the FinchTV version 1.4.0 software (Geospiza, Seattle, WA); the sequences obtained were compared with the reference from GenBank database (NM_023067), and the mutation nomenclature was used according to Human Genome Variation Society guidelines (http://www.hgvs.org/mutnomen). Pathogenic scores for missense mutations were calculated using the prediction tools SIFT, ${ }^{11}$ MutPred, ${ }^{12}$ and MutationTaster. ${ }^{13}$

\section{Genotype-Lacrimal Gland Phenotype Correlation}

To identify a possible genotype-phenotype correlation, 2 groups of mutations were considered: those affecting protein size or its functional domain and those not affecting the protein catalytic domain or its structure. Fisher exact test was used to determine the significance of the association between these 2 categories of mutations and LG abnormalities. A $P$ value $\leq 0.05$ was considered significant.

\section{Results}

Clinical and molecular data are shown in Table 2. Eight of the 21 patients were relatives belonging to 4 distinct families. Patients 1 and 2, 13 and 14, and 15 and 16 were progenitor and offspring, respectively, and patients 18 and 19 were twin brothers. Fifteen patients $(71.4 \%)$ had already been submitted to some surgical procedure to correct their eyelid malformations. In 9 patients (42.8\%), more than 1 procedure had been performed, including multiple revisions of silicone or autologous fascia slings for ptosis surgery. Twenty-two eyes (52.4\%) from 12 patients showed clinical signs of reduced tear production manifested as decreased lacrimal meniscus height, low values on the Schirmer test, or superficial punctate keratopathy (SPK) of variable severity. Of these 22 eyes, 20 (90.9\%) did not have a measurable LG ipsilaterally, and in the remaining 2 eyes, the LG size was markedly reduced.

A significant difference was found between the Schirmer's scores in absent versus reduced LG cases $(P=0.0006)$. Figure 1 shows the distribution of Schirmer's scores versus LG volumes. Eleven of the 14 eyes $(78.6 \%)$ with absent LG showed Schirmer's values below the $10-\mathrm{mm}$ cut off. In the group with reduced LG volumes, the Schirmer's scores were highly variable ranging from 3 to $35 \mathrm{~mm}$. All the patients with normal LG presented normal Schirmer's values. A Schirmer's score less than $10 \mathrm{~mm}$ had a sensitivity of $68.4 \%$ to identify an LG volume of less than $0.20 \mathrm{~cm}^{3}$ (Fig 1). Finally, the Fisher exact test revealed that the presence of SPK was significantly associated with an absent LG $(P=0.0007)$.

Orbital CT scans disclosed LG agenesis in 11 patients, 9 $(42.8 \%)$ bilaterally and 2 unilaterally. Figure 2 shows examples of the morphologic LG variants detected. With the exception of 3 patients (6 glands), all LG volumes were reduced and below the minimum value measured in the control group (Table 2). In regard to only patients with BPES with measurable LG (Fig 3), median LG volumes were $0.22 \mathrm{~cm}^{3}$ (range, $0.06-0.36 \mathrm{~cm}^{3}$ ) and $0.24 \mathrm{~cm}^{3}$ (range, $0.08-0.34 \mathrm{~cm}^{3}$ ) concerning the right and left sides, respectively, with no significant difference between sides $(P=0.72)$. In the control group, median LG volumes were 0.54 $\mathrm{cm}^{3}$ in the right orbits (mean $0.55 \mathrm{~cm}^{3}$; standard deviation, 0.19 ; range, $0.30-1.22$ ) and $0.54 \mathrm{~cm}^{3}$ in the left orbits (mean, 0.53; standard deviation, 0.20; range, 0.32-1.29), values similar to those previously reported in the literature. ${ }^{10}$ The Mann-Whitney test showed that the volumes of patients with BPES were significantly lower than in the control $(P<0.05)$ for both the right and left orbits.

\section{Molecular Investigation}

Molecular screening of the FOXL2 gene showed pathogenic sequence variants in $13(81.2 \%)$ of the 16 patients analyzed (Fig 4; 
Table 2. Clinical, Imaging, and Molecular Analysis of Patients with Blepharophimosis-Ptosis-Epicanthus Inversus Syndrome

\begin{tabular}{|c|c|c|c|c|c|c|c|c|c|c|c|c|}
\hline \multirow[b]{2}{*}{ Patient } & \multirow[b]{2}{*}{$\begin{array}{l}\text { Age } \\
\text { (yrs) }\end{array}$} & \multirow[b]{2}{*}{ Gender } & \multirow[b]{2}{*}{$\begin{array}{l}\text { No. of Previous } \\
\text { Surgeries }\end{array}$} & \multirow[b]{2}{*}{ Laterality } & \multicolumn{3}{|c|}{ Lacrimal Film Evaluation } & \multicolumn{2}{|c|}{ Lacrimal Gland Imaging } & \multicolumn{3}{|c|}{ FOXL2 Molecular Analysis } \\
\hline & & & & & $\begin{array}{l}\text { Lacrimal } \\
\text { Meniscus }\end{array}$ & $\begin{array}{l}\text { Schirmer } \\
I(\mathrm{~mm})\end{array}$ & $\begin{array}{l}\text { Superficial } \\
\text { Keratopathy }\end{array}$ & $\begin{array}{l}\text { Glandular } \\
\text { Volume }\left(\mathrm{cm}^{3}\right)\end{array}$ & $\begin{array}{l}\text { Lacrimal Gland } \\
\text { Size Rating* }\end{array}$ & cDNA & Protein & Type \\
\hline \multirow[t]{2}{*}{1} & 14 & $\mathrm{~F}$ & 1 & $\mathrm{R}$ & + & 18 & $\mathrm{~N}$ & 0.24 & Reduced & c.672_701dup30 & p.A224-A234dup & In-frame duplication \\
\hline & & & & $\mathrm{L}$ & + & 24 & $\mathrm{~N}$ & 0.28 & Reduced & & & \\
\hline \multirow[t]{2}{*}{2} & 38 & $\mathrm{~F}$ & None & $\mathrm{R}$ & + & 15 & $\mathrm{~N}$ & 0.26 & Reduced & c.672_701dup30 & p.A224-A234dup & In-frame duplication \\
\hline & & & & $\mathrm{L}$ & + & 17 & $\mathrm{~N}$ & 0.29 & Reduced & & & \\
\hline \multirow[t]{2}{*}{3} & 11 & F & 3 & $\mathrm{R}$ & $\downarrow$ & 15 & Diffuse & NM & Absent & Not detected & $\mathrm{X}$ & $\mathrm{X}$ \\
\hline & & & & $\mathrm{L}$ & $\downarrow$ & 12 & Diffuse & NM & Absent & & & \\
\hline \multirow[t]{2}{*}{4} & 11 & M & 2 & $\mathrm{R}$ & $\downarrow$ & 2 & Inferior & NM & Absent & Not detected & $\mathrm{X}$ & $\mathrm{X}$ \\
\hline & & & & $\mathrm{L}$ & + & 12 & $\mathrm{~N}$ & 0.24 & Reduced & & & \\
\hline \multirow[t]{2}{*}{5} & 13 & M & 1 & $\mathrm{R}$ & + & 32 & $\mathrm{~N}$ & 0.29 & Reduced & c. $650 \mathrm{C}>\mathrm{G}$ & p.S217C & Missense \\
\hline & & & & $\mathrm{L}$ & + & 35 & $\mathrm{~N}$ & 0.25 & Reduced & & & \\
\hline \multirow[t]{2}{*}{6} & 19 & $\mathrm{~F}$ & 2 & $\mathrm{R}$ & + & 23 & $\mathrm{~N}$ & 0.36 & $\mathrm{~N}$ & Not detected & $\mathrm{X}$ & $\mathrm{X}$ \\
\hline & & & & $\mathrm{L}$ & + & 18 & $\mathrm{~N}$ & 0.34 & $\mathrm{~N}$ & & & \\
\hline \multirow[t]{2}{*}{7} & 10 & $\mathrm{~F}$ & 1 & $\mathrm{R}$ & + & $>35$ & $\mathrm{~N}$ & 0.32 & $\mathrm{~N}$ & c.672_701dup30 & p.A224-A234dup & In-frame duplication \\
\hline & & & & $\mathrm{L}$ & + & $>35$ & $\mathrm{~N}$ & 0.32 & $\mathrm{~N}$ & & & \\
\hline \multirow[t]{2}{*}{8} & 8 & M & 2 & $\mathrm{R}$ & $\downarrow$ & 7 & Inferior & NM & Absent & c. $292 \mathrm{~T}>\mathrm{G}$ & p.Y98G & Missense \\
\hline & & & & $\mathrm{L}$ & $\downarrow$ & 11 & Inferior & NM & Absent & & & \\
\hline \multirow[t]{2}{*}{9} & 3 & $\mathrm{~F}$ & 1 & $\mathrm{R}$ & + & Unc & $\mathrm{N}$ & 0.11 & Reduced & c.172_176delTCGTA & p.S58RfsX36 & In-frame deletion \\
\hline & & & & $\mathrm{L}$ & + & Unc & $\mathrm{N}$ & 0.08 & Reduced & & & \\
\hline \multirow[t]{2}{*}{10} & 2 & F & None & $\mathrm{R}$ & $\downarrow$ & Unc & Unc & NM & Absent & c.919_920insACCGCCGC & p.P307HFsX52 & In-frame insertion \\
\hline & & & & $\mathrm{L}$ & $\downarrow$ & Unc & Unc & NM & Absent & & & \\
\hline \multirow[t]{2}{*}{11} & 9 & M & 2 & $\mathrm{R}$ & $\downarrow$ & 3 & Inferior & 0.17 & Reduced & c. $370 \mathrm{~A}>\mathrm{T}$ & p.K124Stop & Nonsense \\
\hline & & & & $\mathrm{L}$ & $\downarrow$ & 4 & Inferior & 0.12 & Reduced & & & \\
\hline \multirow[t]{2}{*}{12} & 2 & M & None & $\mathrm{R}$ & $\downarrow$ & Unc & Unc & NM & Absent & c.768-769insG & p.P257AFs & In-frame insertion \\
\hline & & & & $\mathrm{L}$ & $\downarrow$ & Unc & Unc & $\mathrm{NM}$ & Absent & & & \\
\hline \multirow[t]{2}{*}{13} & 39 & M & None & $\mathrm{R}$ & $\downarrow$ & 3 & $\mathrm{~N}$ & NM & Absent & c. $313 \mathrm{~A}>\mathrm{C}$ & p.N105H & Missense \\
\hline & & & & $\mathrm{L}$ & $\downarrow$ & 4 & $\mathrm{~N}$ & NM & Absent & & & \\
\hline 14 & 5 & $\mathrm{~F}$ & None & $\mathrm{R}$ & $\downarrow$ & Unc & Unc & NM & Absent & c. $313 \mathrm{~A}>\mathrm{C}$ & p.N105H & Missense \\
\hline & & & & $\mathrm{L}$ & $\downarrow$ & Unc & Unc & NM & Absent & & & \\
\hline 15 & 36 & M & 2 & $\mathrm{R}$ & + & 22 & $\mathrm{~N}$ & 0.12 & Reduced & c.672_701dup30 & p.A224-A234dup & In-frame duplication \\
\hline & & & & $\mathrm{L}$ & + & 32 & $\mathrm{~N}$ & 0.14 & Reduced & & & \\
\hline 16 & 8 & M & 5 & $\mathrm{R}$ & + & Unc & $\mathrm{N}$ & 0.06 & Reduced & c.672_701dup30 & p.A224-A234dup & In-frame duplication \\
\hline & & & & $\mathrm{L}$ & + & Unc & $\mathrm{N}$ & 0.16 & Reduced & & & \\
\hline 17 & 7 & $\mathrm{~F}$ & 1 & $\mathrm{R}$ & + & 18 & $\mathrm{~N}$ & 0.10 & Reduced & Not performed & & \\
\hline & & & & $\mathrm{L}$ & + & 6 & Inferior & NM & Absent & & & \\
\hline 18 & 27 & M & 2 & $\mathrm{R}$ & + & 4 & Inferior & NM & Absent & Not performed & & \\
\hline & & & & $\mathrm{L}$ & $\downarrow$ & 0 & Inferior & NM & Absent & & & \\
\hline 19 & 27 & M & 2 & $\mathrm{R}$ & $\downarrow$ & 0 & Inferior & NM & Absent & Not performed & & \\
\hline & & & & $\mathrm{L}$ & $\downarrow$ & 0 & Inferior & NM & Absent & & & \\
\hline 20 & 31 & $\mathrm{~F}$ & None & $\mathrm{R}$ & NP & NP & $\mathrm{N}$ & 0.32 & $\mathrm{~N}$ & Not performed & & \\
\hline & & & & $\mathrm{L}$ & NP & NP & $\mathrm{N}$ & 0.33 & $\mathrm{~N}$ & & & \\
\hline 21 & 2 & $\mathrm{~F}$ & 1 & $\mathrm{R}$ & $\downarrow$ & 0 & Inferior & NM & Absent & Not performed & & \\
\hline & & & & $\mathrm{L}$ & $\downarrow$ & 8 & $\mathrm{~N}$ & NM & Absent & & & \\
\hline
\end{tabular}




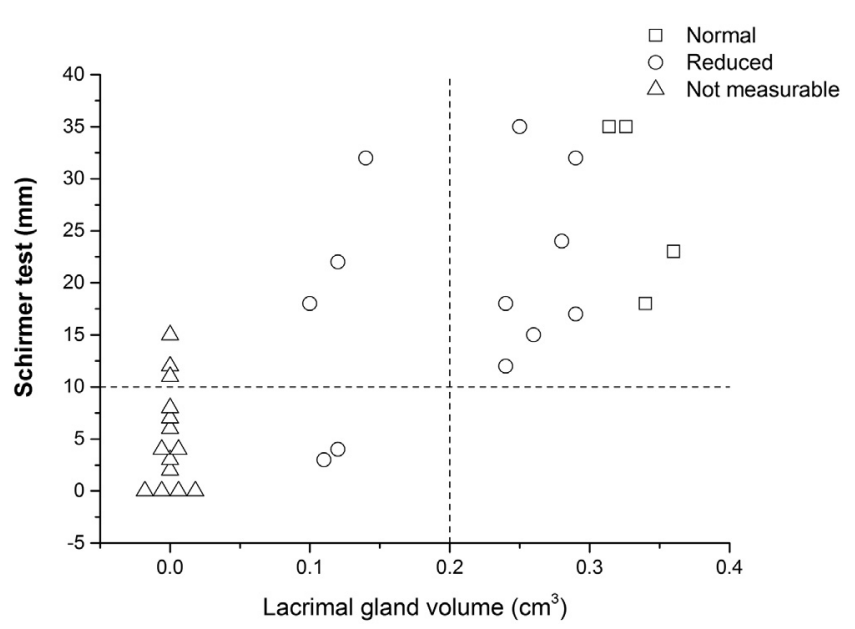

Figure 1. Distribution of Schirmer I test with lacrimal gland (LG) volumes.

Table 2). Eight distinct mutations were found, 4 of them previously unreported.

The most frequent mutation in this study was the in-frame duplication of 30 nucleotides, c.672_701dup30, resulting in a polyalanine expansion (p.A224-A234dup) that was detected in 5 patients (patients 1, 2, 7, 15, and 16) from 3 distinct families. Three missense mutations also were detected (c.650C $>$ G, c.292T $>$ G, c. $313 \mathrm{~A}>\mathrm{C}$ ), the last 2 in the DNA-binding forkhead domain of the gene.

Two in-frame insertions (c.919_920insACCGCCGC and c.768-769insG), a nonsense mutation (c.370A $>$ T), and an in-frame deletion (c.172_176delTCGTA) were also found. All 4 interfered with the stop codon position; 3 of them led to a premature stop codon location (and abnormally small proteins), and the insertion from patient 12 , c.768-769insG, deleted the wild-type stop codon of the gene, producing a hyper-long protein. Mutations c.172_176delTCGTA, c.370A $>$ T, c.768-769insG, and c.313A $>$ C from patients $9,11,12$, and 13/14, respectively, were not previously described in BPES. The methodology used did not show any pathogenic mutations in 3 unrelated patients.

\section{Genotype-Lacrimal Gland Phenotype Association}

Six distinct mutations were associated with a more severe lacrimal phenotype, including absent or significantly reduced LG size and tear hyposecretion. These mutations comprised sequence variants inducing premature stop codons (c.172_176delTCGTA, c.919_920insACCGCCGC, and c.370A $>$ T) or interfering with the wild-type one (c.768-769insG), and missense mutations located in the DNA-binding forkhead domain (c.292T $>$ G, c.313A $>$ C). The Fisher exact test showed a higher probability for these mutations to be associated with agenesis or underdevelopment of the gland $(P=0.021)$.

Lighter phenotypes, with LG volumes within or near control group values and normal tear evaluation, were detected in association with alanine expansions (c.672_701dup30) and a missense mutation just before the polyalanine tract of the FOXL2 gene (c.650C $>\mathrm{G}$ ).

In 3 unrelated patients, the gene sequence was normal. Two of these cases presented bilateral or unilateral LG agenesis (patients 3 and 4 , respectively), and in the remaining one the gland was normal.

\section{Discussion}

The clinical presentation of BPES has been extensively described. ${ }^{14}$ In addition to the classic signs of ptosis, epicanthus inversus and telecanthus, a variety of ophthalmic and nonophthalmic features ${ }^{15-20}$ are variably associated with the syndrome. Deficiency of the tear production apparatus has been overlooked. To our knowledge, there are only 2 case reports of lacrimal hyposecretion. Athappilly and Braverman ${ }^{21}$ described a 9-month-old female patient with BPES and bilateral absence of the LG. More recently, another 9-month-old infant with BPES was found to have absent tear production though normal-sized LGs were visible on ultrasound imaging. ${ }^{22}$

The present report is the first to show that the association between alacrima and BPES is not incidental. In our group of patients, $52.3 \%$ had no measurable LGs $(42.8 \%$ bilaterally and $9.5 \%$ unilaterally), and $33 \%$ of the remaining cases showed reduced LG volume. Not surprisingly, there was a clear association between these radiologic findings and tear hyposecretion.

A genotype-phenotype correlation has not been proven as yet in BPES. ${ }^{23}$ So far, more than 100 different mutations have been described involving FOXL2, and pathogenic variants can be found in approximately $88 \%$ of cases. ${ }^{24}$ The presence of other loci causing the syndrome also has been proposed, although a clear association is still lacking. ${ }^{17,25,26}$ We performed molecular screening of FOXL2 in 16 patients to analyze specifically the LG phenotype. Thirteen patients (81\%) showed pathogenic genetic changes, 4 of them already described by other authors ${ }^{27-30}$ and the remaining 4 being novel mutations. The most frequent mutation was a 30-nucleotide duplication (c.672- 701dup30) causing an expansion of 10 alanine residues (p.A224-234dup) within the polyalanine tract (Fig 4). This mutation is responsible for a shift in the protein location from the nucleus, where it is normally located, to the cytoplasm, and induces a strong tendency to aggregation. $^{31}$ In our study, the same type of alanine duplication was found in 2 distinct families (1 of them Portuguese and the other Brazilian) and in 1 Brazilian sporadic case. This genetic defect is, in fact, recurrent and has been reported in distinct families with different geographic and ethnic backgrounds. ${ }^{27}$

We found 3 missense mutations, 2 of them, c.650C $>\mathrm{G}$ and c.292T $>$ G, already known. The first ${ }^{28}$ leads to a switch from the amino acid serine to cysteine, immediately upstream from the polyalanine domain. This substitution presumably acts as a loss-of-function mutation, producing hypomorphic or null alleles and haploinsufficiency. ${ }^{29}$ The latter is a change from a tryptophan to a glycine within the forkhead domain of FOXL2, a more common location for missense mutations in BPES, and causes nuclear and cytoplasmic protein aggregation. $^{32}$ The third missense mutation $(\mathrm{c} .313 \mathrm{~A}>\mathrm{C})$, found in 2 related patients, is a novel pathogenic variant and causes the substitution of an asparagine for a histidine in the forkhead domain of FOXL2. ${ }^{24}$ The remaining 4 mutations included 2 insertions, c.919_920insACCGCCGC 


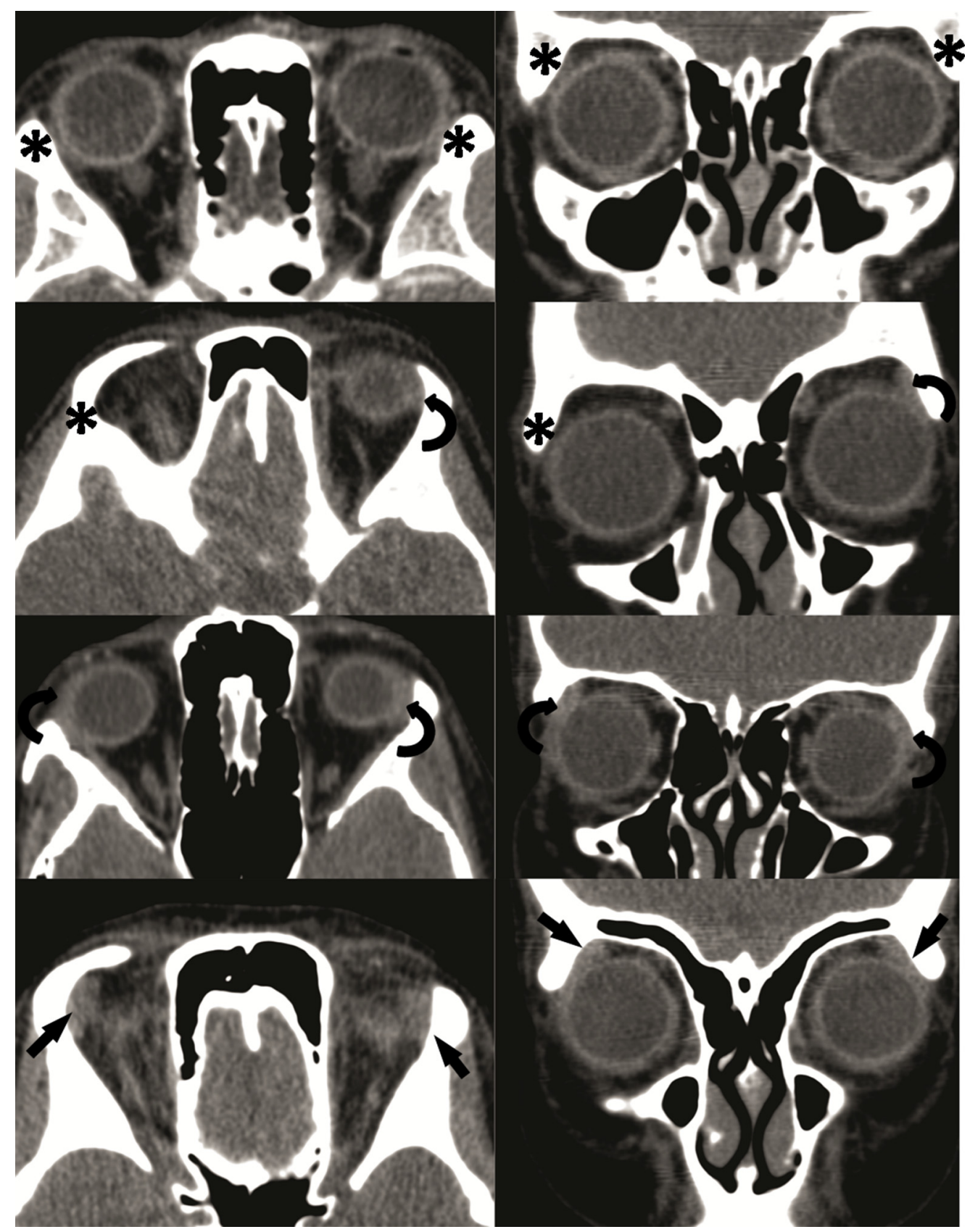

Figure 2. From top to bottom: axial (left) and coronal (right) computed tomography (CT) scans of 4 distinct patients. Asterisks demonstrate absence of the lacrimal gland (LG), curved arrows highlight reduced LG, and straight arrows indicate a normal-sized LG.

and c.768-769insG, a nonsense mutation, c.370A $>$ T, and a deletion, c.172_176delTCGTA. Only the first insertion has been described. ${ }^{30}$

By taking into consideration only the LG phenotype and the mutation data, our data suggest that there is an association between the type of mutation and its location and LG abnormality. This finding needs to be corroborated by the evaluation of larger samples.

Overall, lower Schirmer scores in patients with BPES are highly suspicious for LG underdevelopment or even agenesia. The majority of patients with absent LG presented subnormal Schirmer scores. Nonetheless, 3 eyes with not measurable LG displayed values above the $10-\mathrm{mm}$ cut off. We believe that these higher scores might be the result of the presence of small amounts of lacrimal tissue indistinguishable in the CT scan. The LG is surrounded by numerous structures with different radiologic densities, which make the identification of a small quantity of lacrimal tissue difficult, even for trained radiologists.

In the group of eyes with reduced LG volumes, Schirmer scores were highly variable. One patient with very small LGs (patient 11) presented low scores; however, in the 


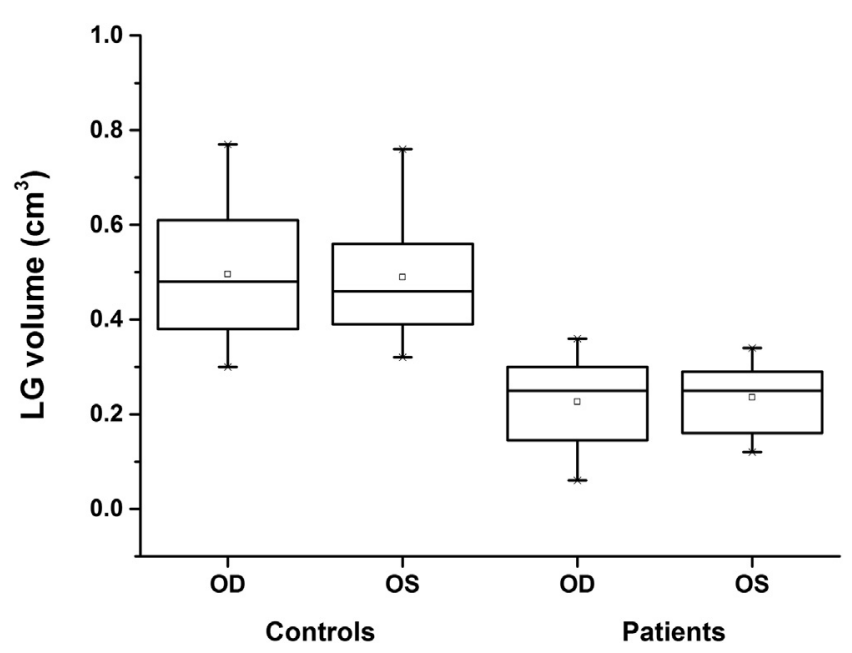

Figure 3. Box-plot diagrams showing the right eye and left eye lacrimal gland $(\mathrm{LG})$ volume distribution in the control group $(\mathrm{n}=30)$ and patients with blepharophimosis-ptosis-epicanthus inversus syndrome (BPES) $(\mathrm{n}=$ 21). The central rectangle represents the interquartile range, the line inside the rectangle represents the median, the small square indicates the mean, and the whiskers above and below the box show the minimum and maximum values. $\mathrm{OD}=$ right eye; $\mathrm{OS}=$ left eye.

remaining eyes there was not a clear relationship between LG size and Schirmer test. Some eyes with near-normal LG size displayed low Schirmer scores. When interpreting these results, one should keep in mind the unreliable nature and high variability of the Schirmer test. ${ }^{33}$ Besides that, our data combined with a previous reported case of alacrima in a child with BPES and normal-sized $\mathrm{LG}^{22}$ might indicate that FOXL2 mutations have an impact not only in the size but also in the gland's function.

Three patients (numbers 6, 7, and 20) displayed normal LG volumes. Schirmer test was performed in 2 of these patients with normal scores. We could not find an association between normal-sized LG and the molecular results. One of the patients (number 6) did not have an identifiable mutation, patient 7 presented the alanine expansion c.672-701dup30, also associated with reduced size LG (Table 2), and in patient 20 the genetic screening was not performed.

The prevalence of LG underdevelopment in BPES remains unknown. In a previous study involving clinical, radiologic, and genetic analysis of 33 Indian patients with BPES, Chawla et $\mathrm{al}^{17}$ used CT scans to study bone development and to compare orbital biometry with age-matched controls. No changes were detected in the LGs, which were reported to be apparently normal. We believe that a more accurate analysis of glandular volumes probably could show significant differences compared with the age-matched control group. However, it is interesting to notice that the rate of intragenic mutations in this group of patients with BPES was only $4 \%(1 / 25)$, which is below the usually reported rate of $72 \%$ in western countries. ${ }^{30}$ As Chawla et $\mathrm{al}^{17}$ suggest, this may have an ethnic reason, with the existence of other locus not yet described responsible for this distinct genetic background.

Considering our results, we must emphasize the importance of LG agenesis for the surgical management of BPES. There is a significant burden for the parents of affected children, who are usually extremely concerned about the

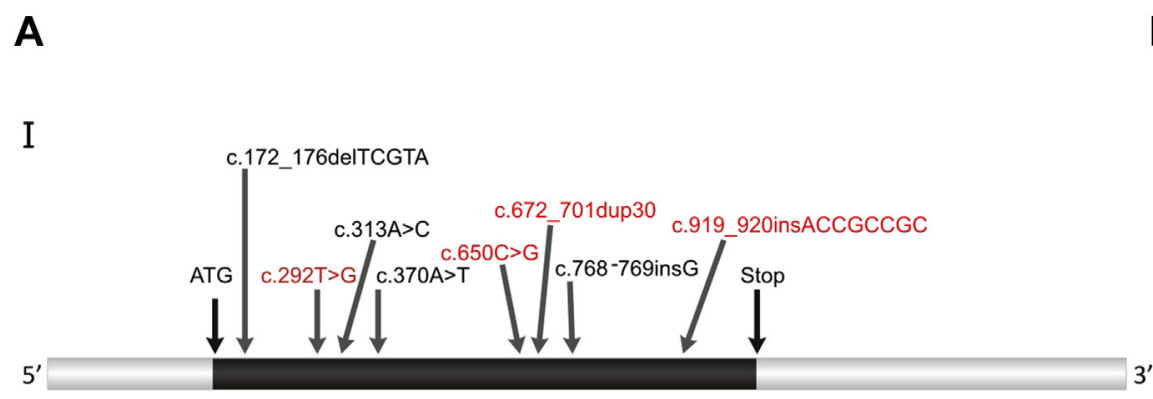

II

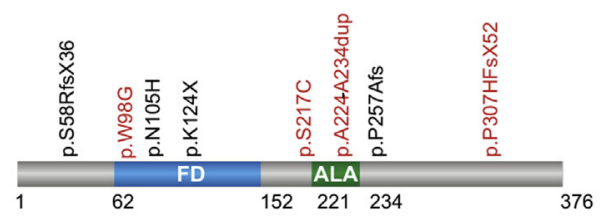

B $1111+1+1$
GCGGCGGGAGCGCTAGG CAACTAC TGG ACGCTGGA

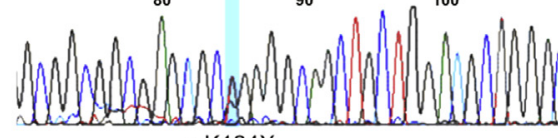
p.K124X AAAATAGCATCCGCCACCACCTCAGCCTCAACGAGT

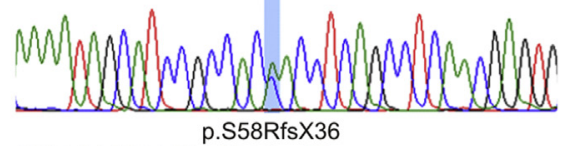

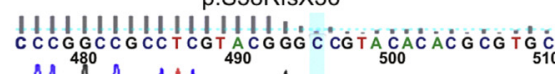

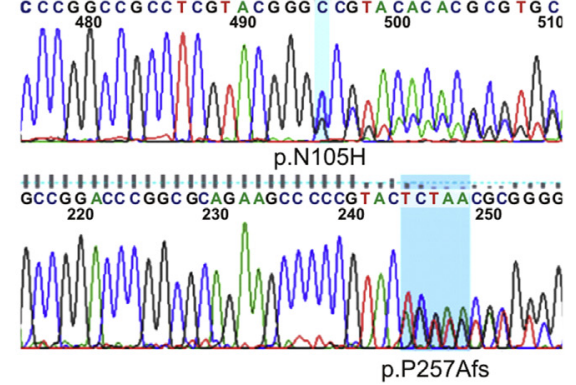

Figure 4. FOXL2 mutations causing blepharophimosis-ptosis-epicanthus inversus syndrome (BPES). A, Organization of the human FOXL2 gene (I) and its protein product (II). Mutations in black = mutations described in the current article; mutations in red = mutations previously described in the literature. Forkhead domain, poly-ALA. B, Representative chromatograms of novel FOXL2 mutations. K124X (nonsense), S58RfsX36 (in-frame 5 amino acid deletion), N105H (missense), and P257Afs (in-frame 1 amino acid insertion). FD = forkhead domain. 
abnormal appearance of the patients and exert a tremendous pressure for early cosmetic rehabilitation. Although there is no consensus about the best surgical strategy for the correction of the multiple palpebral fissure anomalies in BPES, ${ }^{14}$ early ptosis surgery may be needed to alleviate the chin position and avoid visual deprivation. ${ }^{34}$ Ptosis in BPES is generally severe, with poor to zero levator function, and most authors recommend bilateral frontalis slings. Supramaximal levator resection ${ }^{35}$ and frontalis muscle flaps $^{36,37}$ have been attempted with variable results. For these reasons, a reduced tear production is a serious concern for the long-term effects of ptosis correction on the ocular surface, because it seems reasonable to accept that spontaneous blink amplitude might be reduced after all these different modalities of surgery. ${ }^{38}$ In addition, a certain degree of nocturnal lagophthalmos is the rule after congenital ptosis surgery, increasing the odds of corneal complications. In our cohort, all patients with demonstrable LG absence had persistent SPK; however, we cannot ignore the influence of postsurgical lagophthalmos in this corneal staining.

In conclusions, tear film evaluation often is neglected in children with BPES mainly because of the low position of the upper lid and the lack of cooperation. Nevertheless, our findings support the need for a careful preoperative evaluation of the lacrimal production status in all patients with BPES. If there is any sign of SPK or a history of tear absence when crying, orbital imaging should be considered to confirm LG underdevelopment.

Acknowledgments. The authors thank Thais Oliveira dos Anjos and Adriana Aparecida Marques for technical support. The authors also acknowledge the cooperation of patients and parents, and the physicians who provided patients' clinical data and samples, namely, Gherusa Moré Sahar and Marcelo Goldberg.

\section{References}

1. Vignes M. Epicanthus héréditaire. Recueil d'Ophthalmologie. 1989:422-425.

2. Dimitry TJ. Hereditary ptosis. Am J Ophthalmol. 1921;45: 655-658.

3. Crisponi L, Deiana M, Loi A, et al. The putative forkhead transcription factor FOXL2 is mutated in blepharophimosis/ ptosis/epicanthus inversus syndrome. Nat Genet. 2001;27: $159-166$.

4. Cocquet J, De Baere E, Gareil M, et al. Structure, evolution and expression of the FOXL2 transcription unit. Cytogenet Genome Res. 2003;101:206-211.

5. Heude E, Bellessort B, Fontaine A, et al. Etiology of craniofacial malformations in mouse models of blepharophimosis, ptosis and epicanthus inversus syndrome. Hum Mol Genet. 2015;24:1670-1681.

6. Zlotogora J, Sagi M, Cohen T. The blepharophimosis, ptosis, and epicanthus inversus syndrome: delineation of two types. Am J Hum Genet. 1983;35:1020-1027.

7. De Baere E, Copelli S, Caburet S, et al. Premature ovarian failure and forkhead transcription factor FOXL2: blepharophimosis-ptosis-epicanthus inversus syndrome and ovarian dysfunction. Pediatr Endocrinol Rev. 2005;2:653-660.
8. Shapiro A, Merin S. Schirmer test and break-up time of tear film in normal subjects. Am J Ophthalmol. 1979;88: 752-757.

9. Serin D, Karsloglu S, Kyan A, Alagoz G. A simple approach to the repeatability of the Schirmer test without anesthesia: eyes open or closed? Cornea. 2007;26:903-906.

10. Bingham CM, Castro A, Realini T, et al. Calculated CT volumes of lacrimal glands in normal Caucasian orbits. Ophthal Plast Reconstr Surg. 2013;29:157-159.

11. Kumar P, Henikoff S, Ng PC. Predicting the effects of coding non-synonymous variants on protein function using the SIFT algorithm. Nat Protoc. 2009;4:1073-1081.

12. Li B, Krishnan VG, Mort ME, et al. Automated inference of molecular mechanisms of disease from amino acid substitutions. Bioinformatics. 2009;25:2744-2750.

13. Schwarz JM, Rodelsperger C, Schuelke M, Seelow D MutationTaster evaluates disease-causing potential of sequence alterations. Nat Methods. 2010;7:575-576.

14. Allen CE, Rubin PA. Blepharophimosis-ptosis-epicanthus inversus syndrome (BPES): clinical manifestation and treatment. Int Ophthalmol Clin. 2008;48:15-23.

15. Choi KH, Kyung S, Oh SY. The factors influencing visual development in blepharophimosis-ptosis-epicanthus inversus syndrome. J Pediatr Ophthalmol Strabismus. 2006;43:285-288.

16. Dawson EL, Hardy TG, Collin JR, Lee JP. The incidence of strabismus and refractive error in patients with blepharophimosis, ptosis and epicanthus inversus syndrome (BPES). Strabismus. 2003;11:173-177.

17. Chawla B, Bhadange Y, Dada R, et al. Clinical, radiologic, and genetic features in blepharophimosis, ptosis, and epicanthus inversus syndrome in the Indian population. Invest Ophthalmol Vis Sci. 2013;54:2985-2991.

18. Garden JW. Blepharophimosis, ptosis, epicanthus inversus and lacrimal stenosis. Am J Ophthalmol. 1969;67:153-154.

19. Kohn R. Additional lacrimal findings in the syndrome of blepharoptosis, blepharophimosis, epicanthus inversus, and telecanthus. J Pediatr Ophthalmol Strabismus. 1983;20:98100.

20. Cunniff C, Curtis M, Hassed SJ, Hoyme HE. Blepharophimosis: a causally heterogeneous malformation frequently associated with developmental disabilities. Am J Med Genet. 1998;75:52-54.

21. Athappilly GK, Braverman RS. Congenital alacrima in a patient with blepharophimosis syndrome. Ophthalmic Genet. 2009;30:37-39.

22. Ng JK, Stout AU, Aaby AA, Ng JD. Blepharophimosis syndrome with absent tear production. Ophthal Plast Reconstr Surg. 2015;31:e62.

23. De Baere E, Beysen D, Oley C, et al. FOXL2 and BPES mutational hotspots, phenotypic variability, and revision of the genotype-phenotype correlation. Am J Hum Genet. 2003;72: 478-487.

24. Beysen D, De Paepe A, De Baere E. FOXL2 mutations and genomic rearrangements in BPES. Hum Mutat. 2009;30: $158-169$

25. Maw M, Kar B, Biswas J, et al. Linkage of blepharophimosis syndrome in a large Indian pedigree to chromosome 7p. Hum Mol Genet. 1996;5:2049-2054.

26. Zhou L, Wang T, Wang J. Blepharophimosis ptosis epicanthus inversus syndrome with congenital hypothyroidism and brachydactyly in a 7-year-old girl. Ophthal Plast Reconstr Surg. 2016 Apr 25 [Epub ahead of print].

27. Vincent AL, Watkins WJ, Sloan BH, Shelling AN. Blepharophimosis and bilateral Duane syndrome associated with a FOXL2 mutation. Clin Genet. 2005;68:520-523. 
28. Wang J, Liu J, Zhang Q. FOXL2 mutations in Chinese patients with blepharophimosis-ptosis-epicanthus inversus syndrome. Mol Vis. 2007;13:108-113.

29. De Baere E, Dixon MJ, Small KW, et al. Spectrum of FOXL2 gene mutations in blepharophimosis-ptosis-epicanthus inversus (BPES) families demonstrates a genotype-phenotype correlation. Hum Mol Genet. 2001;10:1591-1600.

30. Beysen D, De Jaegere S, Amor D, et al. Identification of 34 novel and 56 known FOXL2 mutations in patients with Blepharophimosis syndrome. Hum Mutat. 2008;29:E205-E219.

31. Caburet S, Demarez A, Moumne L, et al. A recurrent polyalanine expansion in the transcription factor FOXL2 induces extensive nuclear and cytoplasmic protein aggregation. $J$ Med Genet. 2004;41:932-936.

32. Beysen D, Moumne L, Veitia R, et al. Missense mutations in the forkhead domain of FOXL2 lead to subcellular mislocalization, protein aggregation and impaired transactivation. Hum Mol Genet. 2008;17:2030-2038.

33. Nichols KK, Mitchell GL, Zadnik K. The repeatability of clinical measurements of dry eye. Cornea. 2004;23:272-285.
34. Beckingsale PS, Sullivan TJ, Wong VA, Oley C. Blepharophimosis: a recommendation for early surgery in patients with severe ptosis. Clin Experiment Ophthalmol. 2003;31:138142.

35. Decock CE, Shah AD, Delaey $\mathrm{C}$, et al. Increased levator muscle function by supramaximal resection in patients with blepharophimosis-ptosis-epicanthus inversus syndrome. Arch Ophthalmol. 2011;129:1018-1022.

36. Song X, Jia R, Zhu H, et al. A modified staged surgical intervention for blepharophimosis-ptosis-epicanthus inversus syndrome: 125 cases with encouraging results. Ann Plast Surg. 2015;74:410-417.

37. Liu H, Shao Y, Zhao Z, Zhang D. One-stage correction of blepharophimosis-ptosis-epicanthus inversus syndrome using a frontalis muscle transfer technique. J Plast Surg Hand Surg. 2014;48:74-79.

38. Cruz AA, Akaishi PM, Mendonca AK, et al. Supramaximal levator resection for unilateral congenital ptosis: cosmetic and functional results. Ophthal Plast Reconstr Surg. 2014;30: 366-371.

\section{Footnotes and Financial Disclosures}

Originally received: September 21, 2016.

Final revision: October 25, 2016.

Accepted: October 26, 2016.

Available online: December 1, 2016.

Manuscript no. 2016-382.

${ }^{1}$ Division of Oculoplastic and Orbit Surgery, Department of Ophthalmology, Otorhinolaryngology and Head and Neck Surgery, School of Medicine of Ribeirão Preto, University of São Paulo, Ribeirão Preto, São Paulo, Brazil.

${ }^{2}$ Department of Genetics, School of Medicine of Ribeirão Preto, University of São Paulo, São Paulo, Brazil.

${ }^{3}$ Department of Radiology, School of Medicine of Ribeirão Preto, University of São Paulo, Ribeirão Preto, São Paulo, Brazil.

${ }^{4}$ Division of Pediatric Ophthalmology and Strabismus, Centro Hospitalar de Lisboa Central, Lisbon, Portugal.

Financial Disclosure(s):

The author(s) have no proprietary or commercial interest in any materials discussed in this article.
Author Contributions:

Conception and design: Duarte, Cruz

Data collection: Duarte, Akaishi, Chodraui-Filho, Cintra, Toscano, Silva Jr. Analysis and interpretation: Duarte, de Molfetta, Chodraui-Filho, Cintra, Toscano, Silva Jr.

Obtained funding: Not applicable

Overall responsibility: Duarte, Cruz

Abbreviations and Acronyms:

BPES = blepharophimosis-ptosis-epicanthus inversus syndrome; CT $=$ computed tomography; FOXL2 $=$ forkhead box protein L2; $\mathbf{L G}=$ lacrimal gland; $\mathbf{S P K}=$ superficial punctate keratopathy.

Correspondence:

Antonio A.V. Cruz, MD, Department of Ophthalmology, Otorhinolaryngology and Head and Neck Surgery, Hospital das Clínicas-Campus, School of Medicine of Ribeirão Preto, University of São Paulo, Ribeirão Preto, 14049-900 São Paulo, Brazil. E-mail: aavecruz.fmrp@gmail.com. 